ISSN 2693-2504

\title{
Design and Analysis of Mechanical Microswimmers
}

Journal of Bioscience \& Biomedical Engineering

Research Article

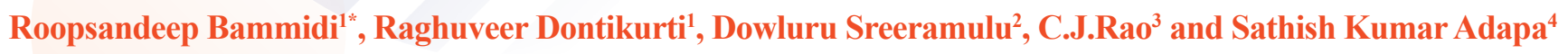

*Correspondence authors

\author{
${ }^{1 * 1,4}$ Assistant Professor, Department of Mechanical \\ Engineering, Aditya Institute of Technology and Management, \\ Tekkali, India. \\ 2,3 Professor, Department of Mechanical Engineering, Aditya \\ Institute of Technology and Management, Tekkali, India.
}

\author{
Roopsandeep Bammidi \\ Assistant Professor \\ Department of Mechanical Engineering \\ Aditya Institute of Technology and Management \\ Tekkali \\ India
}

Submitted : 16 Jul 2021 ; Published : 4 Nov 2021

\begin{abstract}
The fascinating field in present civilization scenario at the edge of science is micro-swimmers, which is a combination of bio physics with self-propulsion mechanisms involving swimming strategies at low Reynolds number. These micro swimming robots offer many advantages in biomedical applications such as drug delivery to some specific locations in our human body and also conducting some surgical operations like opening of blocked arteries etc. In recent times, blocked arteries become a major case in the medical world. This is can be diagnosed by Angioplasty (is a minimally invasive, endovascular procedure to widen narrowed or obstructed arteries or veins, typically to treat arterial atherosclerosis) method. So this is the main reason to choose the aorta as our domain for analysis purpose. This paper presents a micro-swimmer with three different heads they are spherical head, Capsule type head and Tapered cylindrical or elliptical head and modelled using SOLIDWORKS and analysis in ANSYS FLUENT.
\end{abstract}

Keywords: Micro swimmers, Solidworks, Ansys Fluent, Self-Propulsion, Micro-Robots.

\section{Introduction}

Micro-swimmers are the bio-hybrid micro-robots consisting of artificial microstructures. It is designed mechanically with selfpropulsion. This is the application of engineering principles and technology to medicine and biology for healthcare purposes. In the present situation of medical life, it is necessary to close the gap between engineering and medicine for advanced health care treatment. Micro-swimmer is the next step for that, having wide range of applications. It is able to travel in the human body through the Arteries, and then it can treat the entire body internally and can give the information and report of every part of the body. As arteries of a human body looks like a thin wire, then the micro-swimmer which will be induced in it should be in micro level. So here the design of micro-swimmer matters very important and it is very complicated. Hence our main agenda is design, analysis and dynamics of mechanical micro-swimmer. Tiny swimmers, be they micro-organisms or micro-bots, live in a world dominated by friction. In this world, technically, the world of low Reynolds numbers, motion is associated with energy dissipation. In the absence of external energy supply objects rapidly come to rest. It is both conceptually interesting, and technologically important, for example in applications to nano-medicine, to try and understand what classes of strategies lead to effective swimming in a setting dominated by dissipation. A particularly promising class of strategies is where the motion is, in a sense, only apparent; where a shape moves with little or no motion of material particles.
Artificial Micro-/ Nano-robots have attracted lots of researchers to carry on extensive study due to their considerable promise for diverse biomedical tasks such as targeted therapy, tissue removal and micro-manipulation. Purcell found the advantages of the nonreciprocal motion and demonstrated two efficient swimming modes at low Reynolds number the flexible oar and the corkscrew. Although Qiuetal presented a micro-swimmer that moves with reciprocal periodic body-shape changes in non-Newtonian fluids, its propulsion performance depended on the fluid viscosity upon varying the shear rate. Recently, more attention has been focus on helical magnetic micro-swimmers which can convert the rotation energy along their helical axis into the translation energy actuated by the uniform magnetic field. Generally speaking, there are two major physical mechanisms to generate a propulsion force in the small scale to overcome the drag force exerted by the surrounding liquid: "inertia propulsion" and "viscous propulsion". The inertia propulsion generates displacements of mass to exchange momentum between the swimming body and the surrounding fluid. The viscous propulsion uses viscous stresses with the surrounding fluid to generate a propulsion force. To classify these two propulsion mechanisms, the Reynolds number (Re), which stands for the ratio between the inertia force and the viscosity force, is commonly used. The Reynolds number is defined by $U L / v$, where $U$ is the characteristic velocity (can be the propulsion speed or actuator speed), L the characteristic physical dimension (swimming body size or actuator size) and $\mathrm{v}$ the kinematic viscosity of the fluid. For the large Re, 
the inertia force is dominantly responsible for propulsion while for the small Re the viscous force is responsible. As the physical dimension critically affects $\mathrm{Re}$, a suitable propulsion mechanism should be selected and used based on the size of the propulsion object or actuator. The locomotion of microscopic organisms, such as bacteria, has been a topic of fascination and mathematical analysis for well over sixty years.

\section{Origin of micro-swimmers}

Apart from seeking fundamental knowledge about how these evolutionarily simple organisms move and interact with their environment, there has recently been great interest in mimicking or using bacteria as micro-robots with biomedical and environmental monitoring applications. Of particular interest are magneto tactic bacteria, which can readily be steered by applying magnetic fields. Organisms that are not naturally magneto tactic can also be controlled with magnetic fields after incorporation of magnetic particles; this has recently been demonstrated with the alga Chlamydomonas reinhardtii. Commonly studied magneto tactic bacteria include the strains MO-1 and MC-1 (Magneto coccus marinus), which are similar in morphology and differ from the species most widely studied in other contexts, such as Escherichia coli, Bacillus subtilis, or Vibrio alginolyticus. For in vivo biomedical applications, micro-swimmers need to find an obstacle-free path and make themselves along the planned path, to accomplish the tasks of targeted therapy and targeted drug delivery. For manual operation, this would allow for considerable time and skilled operators. The manual control method is an open-loop teleoperation without any feedback. Related studies have been reported in the literature for different operation spaces, such as operation in a micro-fluidic chip in $3 \mathrm{D}$ space, and in vivo operation and for different driving methods, such as bacteriadriven operation and chemically driven operation. The manual control method is not appropriate for tasks that require high repetition with high precision.

Motion of micro-swimmer: Micro swimming robots offer many advantages in biomedical applications, such as delivering potent drugs to specific locations in targeted tissues and organs with limited side effects, conducting surgical operations with minimal damage to healthy tissues, treatment of clogged arteries, and collecting biological samples for diagnostic purposes. Reliable navigation techniques for micro swimmers need to be developed to improve the localization of robots inside the human body in future biomedical applications In order to estimate the dynamic trajectory of magnetically propelled micro swimmers in channels, that mimic blood vessels and other conduits, fluid-micro robot interaction and the effect of the channel wall must be understood well. In this study, swimming of one-link robots with helical tails is modeled with Stokes equations and solved numerically with the finite element method. Forces acting on the robot are set to zero to enforce the force-free swimming and obtain forward, lateral and angular velocities that satisfy the constraints. Effects of the number of helical waves, wave amplitude, relative size of the cylindrical head of micro swimmer and the radial position on angular and linear velocity vectors of micro swimmer are presented.
The hydrodynamic characteristics of micro-swimmers can be achieved by the step wise analysis in the Ansys-fluent by adding corresponding values of flow medium(blood).Blood is a body fluid in humans and other animals that delivers necessary substances such as nutrients and oxygen to the cells and transports metabolic waste products away from those cells. It has some standard values like velocity, pressure, density, etc., by providing these data, the velocity vector curves and boundary layer analysis of micro-swimmers can be developed. These swimmers actually a size of microscopic level and therefore the body may not feel the movement of this microswimmer in the arteries. Many of the researches showing that micro-swimmers can have the self propulsion capability when fabricated with a combination of mechanical and electrical devices to give them self actuation and then motion, but this will be very difficult to fabricate such complex structures in the micro size swimmer. So these micro-swimmers are preferable to be worked by an external agent. The most preferable media to make them move is the magnetic field. Magnetic properties can be given to the swimmer and in the presence of a magnetic field it will move. The head of micro-swimmer should be made with a magnetic material. We have different ferromagnetic materials available on our earth. We select the best material from these different materials with the in-toxic and high magnetic strength. The helical tail also has to be made with some magnetic materials. GaAs is the material mostly used to fabricate the tail of the micro-swimmer. This tail is to be attached to a soft nickel spherical body on one side, authors demonstrated the forward motion of the structure in the direction of the helical axis by applying a rotational magnetic field in that direction and pointed out that linear swimming velocity was affected not only by the size of the magnetic head, but also the strength of the applied magnetic field. Cobalt is another ferromagnetic material used for the fabrication of the microswimmer head deposited on one side of the micro-swimmer. Varying the magnetic field strength can give the movement to the micro-swimmer. The blood properties should not change with the magnetic material used. The research journals have been showing that these micro-swimmers first experimented in the water and then by correlating the properties of blood with water.

\section{Classification of micro-swimmers}

There are different types of heads of micro-swimmers but all of these may not show same hydrodynamic characteristics in the fluid media. The properties may change due to the shape of head and tail as they are the only reason for the propulsion. Universally accepted models are solid spherical head and the sperm head. According to the flow and velocity of blood, we should concern the design of head. The head should be structurally tough enough and have a suitable shape for the propulsion. So we considered three types of heads for analysis and simulation. They are spherical head, sperm head and cylindrical head are shown in figure 1-3. 


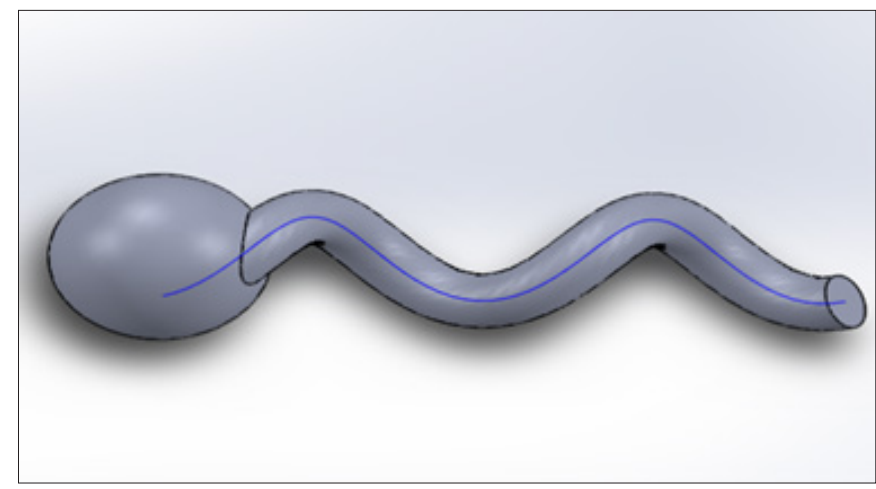

Figure 1: Spherical Head Micro swimmer

The spherical head micro-swimmer design is not a complicated task compared to other designs. Geometry of this microswimmer can make ease of swimming through any fluid media. This is the foremost design used in any application. As its head is spherical, the propulsion in the fluid is very smooth. When the fluid strikes the head on the front part, boundary layer forms around the sphere and pushes the swimmer forward. Hence the swimmer moves easily with this technique. The second design has a band head with helical tail. It looks like a capsule, so named after it. It is a basic three dimensional geometric shape consisting of a cylinder with hemispherical ends. Another name for this shape is spherocylinder. This shape is usually used for containers for pressurized gases and for pharmaceutical capsules. Comparing with other designs, it has more space for accommodation of extra payload.

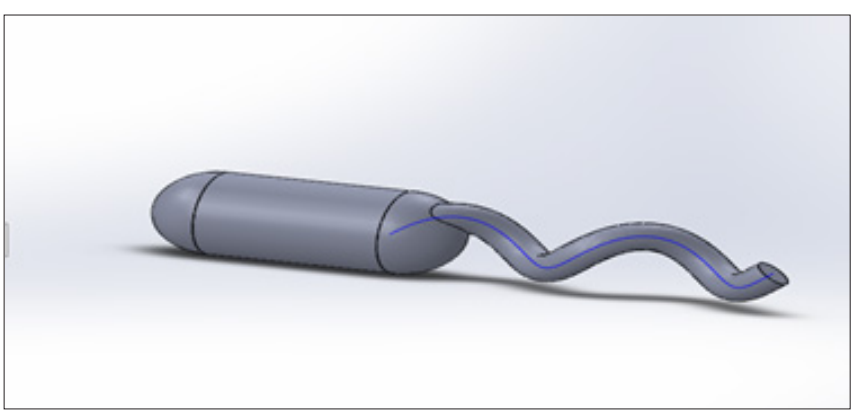

Figure 2: Capsule Head Micro swimmer

This design also is somewhat better for swimming technique, as its ends are semi spherical. Hard sphero cylinders provide a good model for colloidal particles with short ranged repulsive interactions.

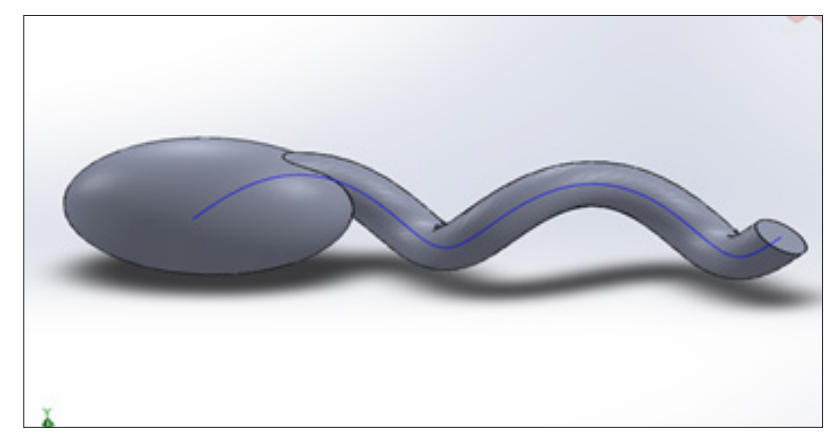

Figure 2: Ellipse Head Micro swimmer
The third design is sperm like head micro-swimmer with a helical tail. This is the most efficient design in any fluid dynamics having exceptional hydrodynamic characteristics compared to other two designs. This design used in every application like, the cockpit of an aeroplane, design of bullet shape, foremost part of a rocket. This design is also can be named as aerofoil shape as it have less drag forces compared to other designs. This is the basic ideal design taken from the sperms of living beings. Apart from the individual characteristics, these are the mostly used designs almost in all research journals, so we considered these as the reference models for our analysis of hydrodynamic characteristics.

\section{Relation of parameters}

In physics and engineering, fluid dynamics is a sub discipline of fluid mechanics that describes the flow of fluids- liquids and gases. It has several sub disciplines, including aerodynamics and hydrodynamics. Fluid dynamics has a wide range of applications, including calculating forces and moments on aircrafts, determining the mass flow rate of petroleum through pipelines, predicting weather patterns. The fluid dynamic analysis can give the information about how the hydrodynamic forces and other parameters act on the bodies when they tend to move or when placed at a position in a fluid flow. In fluid dynamics, drag (sometimes called fluid resistance) is a force, acting opposite to the relative motion of any object moving with respect to a surrounding fluid. This can exists between two fluid layers or between a solid and a fluid layer. Unlike other resistive forces, such as dry friction, which are nearly independent on velocity, drag force depends on velocity. Drag force is proportional to the velocity for a laminar flow and the squared velocity for a turbulent flow. There are different types of drag 1.parastic drag 2.form drag 3.skin friction 4.interference drag 5.lift induced drag 6.wave drag. Drag mainly depends on the shape, size, and speed of the object.

One way to express the drag force is

$$
F_{D}=\frac{1}{2} \rho v^{2} C_{D} A
$$

Here,

$\mathrm{F}$ - is the drag force,

$\mathrm{D}$ - is the density of the fluid

$\mathrm{V}$ - is the speed of the object relative to the fluid,

$\mathrm{A}$ - is the cross sectional area and

Symbol rho - is the drag coefficient - a dimensionless number. The drag coefficient depends on the shape of the object and on the Reynolds number.

$$
R_{e}=\frac{v D}{\nu},
$$

Here $\mathrm{D}$ is some characteristic diameter or linear dimension and $\mathrm{v}$ is the kinematic viscosity of the fluid. At high Reynolds number the drag cofficient is more or less constant and drag will vary as the square of the speed. These are the parameters that effect the motion of a microswimmer in the fluid medium.

Swimming micro-robots have great potential in biomedical applications such as targeted drug delivery, medical diagnosis, 
and destroying blood clots in arteries. Inspired by swimming microorganisms, micro-robots can move in bio-fluids with helical tails attached to their bodies. In order to design and navigate micro-robots, hydrodynamic characteristics of the flow field must be understood well. This work presents computational fluid dynamics modeling and analysis of the flow due to the motion of micro-robots that consist of magnetic heads and helical tails inside fluid-filled channels akin to bodily conduits; special emphasis is on the effects of the radial position of the robot. Time-averaged velocities, forces, torques, and efficiency of the micro-robots placed in the channels are analyzed as functions of rotation frequency, helical pitch (wavelength) and helical radius (amplitude) of the tail. Results indicate that robots move faster and more efficiently near the wall than at the center of the channel. Forces acting on microrobots are asymmetrical due to the chirality of the robot's tail and its motion. Moreover, robots placed near the wall have a different flow pattern around the head when compared to incenter and unbounded swimmers. According to simulation results, time-averaged forward velocity of the robot agrees well with the experimental values measured previously for a robot with almost the same dimensions. Some of the references taken for the future study and analysis of hydrodynamic characteristics of micro-swimmers are as follows:

Gray and Hancock et al. [1] modelled swimming of a seaurchin spermatozoa based on the fluid forces calculated by the resistive force theory, which offers a general framework for the calculation of the resultant propulsion and drag forces from the integration of local forces in normal and tangential directions that are proportional to the velocity components in those directions over the tail. Berke et al. [2] investigated hydrodynamic interactions of swimming organisms with solid surfaces by measuring the distribution of E. coli swimming between glass plates and compared their results with a hydrodynamic model. Najafi et al. [3] proposed another model with linearly linked three spheres for a micro robot that is operated based on the periodical internal motion. According to the numerical study, this swimmer could also work in the low Reynolds number range. Howse et al. [4] coated half of the polystyrene sphere (diameter $1.62 \mu \mathrm{m}$ ) with platinum. When this particle was placed in a hydrogen peroxide solution, it was propelled by breaking hydrogen peroxide into oxygen and water. In a short time period, the swimming was controlled by the hydrogen peroxide concentration at the speed of several $\mu \mathrm{m} / \mathrm{s}$. However, in a long time period, the propelling motion was random. This propulsion mechanism is not clearly understood yet as of today, though there have been two explanations introduced: the osmotic propulsion model and the bubble detachment propulsion model.

Dr. dieter breitschwerdt et al. [5] From a physical point of view, in particular the motion of microorganisms has attracted a lot of attention [12]. Their motion at small scales is mainly governed by viscous forces which dominate over inertial forces, quantified by a small Reynolds number. At low Reynolds numbers microswimmers have to perform non-reciprocal deformations of their cell body in order to swim in the absence of external forces and torques. Bradely j.Nelson et al. [6] —Wireless magnetic micro-robots show great potential for targeted drug delivery or as minimally invasive surgical tools in the human body. In order to swim through bodily fluids, such as the vitreous humor in the eye, they must be equipped to successfully move through viscoelastic fluids, where they are obstructed by fibrous networks or microparticles. Prior researchers have shown an increased propulsion efficiency with increasing viscoelastic properties for artificial helical swimmers and bacteria with helical flagella. Zhang et al. and Peyer et al. [7] proposed Magnetic helical microrobots, powered by external rotating magnetic fields. These microscopic helical swimmers are inspired by the corkscrew motion of bacteria flagella, such as Escherichia coli or Borrelia burgdorferi. They can perform 3-dimensional (3D) navigation in various liquids under low-strength rotating magnetic fields $(<10 \mathrm{mT})$. A. D. Gilbert etal. [8] One of the simplest magnetically based swimming devices realised in the laboratory consists of two ferromagnetic beads joined by an elastic element which could be a vesicle, polymer or protein fibre. The elastic element could also be the component to be transported in a possible medical application. Rotational motion is driven by the torque from an external field. At the same time the direction of the external field and the magnetic anisotropy of the particles influences the directions of the individual ferromagnetic or 'soft' magnets and the dipole force between them, driving a radial component of motion.

Lauga et al. [9] Many microorganisms swim close to boundaries, and as a result the effect of boundaries on fluidbased locomotion has been extensively studied. E. coli bacteria display circular trajectories near boundaries, clockwise when the wall is rigid and anti-clockwise near a free surface. Bernard Bonnard et al. [10] Micro-swimmers were popularized by the seminal presentation and were analyzed very recently using optimization techniques in a series of articles, motivated in particular by industrial applications in robotics to design microrobots whose control is based on the swimming mechanisms of biological micro-swimmers. In particular are sent model was proposed into analyze the observed motions of an abundant variety of zooplanktons called copepods. Francisco Alarcon et al. [11] Micro-swimmers in 3D can generate coordinated response, such as a tendency to swim along each other, or create giant density fluctuations induced by the emergence of a percolating dynamic cluster. We have found that the key factor to produce these collective motions is the hydrodynamic signature of the micro-swimmers. Since the set-up of many experiments is a suspension where particles can move in a quasi 2D geometry, we developed a systematic numerical study such that experimental parameters are simulated.

Joost de Graaf et al. [12] Computationally, hydrodynamic aspects of micro-swimmer suspensions have been studied using a variety of fluid dynamical solvers, including Stokesian dynamics, multi particle collision dynamics (MPCD) boundary element methods and lattice-Boltzmann (LB) simulation. Treating Stokes flow has great advantages from a theoretical point of view but is often difficult to achieve in simulations. 
Methods such as LB and MPCD are constructed to solve the full Navier-Stokes equation, including the inertial term. Adam Wysocki et al. [13] both in a natural environment and in micro-fluidic devices, micro-swimmers usually do not swim in straight but rather in curved or branching micro-channels. Therefore, the influence of surface curvature on accumulation of micro-swimmers is of great interest. For example, a polar micro-swimmer close to a flat wall behaves similarly to a polar particle near a concave surface (e.g., a cavity). This is of high relevance for the design micro-swimmers with controlled walladhesion properties. U Kei Cheang et al. [14] The rotating flagellum forms a helical traveling wave so it is not a reciprocal motion. Various artificial helical swimmers have achieved controlled propulsion under magnetic rotation. The archetypal flexible strategy is the sperm flagellum. Nonreciprocal traveling waves propagate down the flexible flagellum to produce propulsion even for in-plane (hence achiral) beating patterns. Kathrin E. Peyer et al. [15] In nature, micro-organisms have found numerous ways to propel themselves, including beating flexible flagella and cilia. Bacterial swimmers, such as the extensively researched E.coli bacterium, use a molecular motor to rotate helically shaped flagella. The continuous rotation of a helix is a non-reciprocal motion and, therefore, perfectly suited for low Reynolds number navigation. J E Avron et al. [16] the self-locomotion of an elongated micro-swimmer by virtue of unidirectional tangential surface treadmilling is investigated. We show that the propulsion could be almost frictionless, as the micro-swimmer is propelled forward with the speed of the backward surface motion, i.e. it moves through an almost quiescent fluid. We investigate this swimming technique using the special spheroidal coordinates. Dr. Ignacio Pagonabarraga et al. [17] Using kinematics to reveal internal properties of deformable objects moving in fluids is a difficult and longstanding topic in mechanics. One can, for example, think about the motion of a deformable cell in shear flow where various dynamical modes are observed depending on elastic capillary number internal properties of red-blood cells or capsules in a creeping flow. Inferring internal mechanical properties has indeed already being pursued for flexible capsules from their deformation within a flow in a number of contributions. $\mathrm{O}$. Kenneth et al. [18] Swimming at low Reynolds numbers can be remote from common intuition because of the absence of inertia. In fact, even the direction of swimming may be hard to foretell. At the same time, and not unrelated to this, it does not require elaborate designs: Any stroke that is not self retracing will, generically, lead to some swimming. Martel et al. [19] Manufactured a helical filament with dimensions of $1.8 \mathrm{~lm}$ in width, $30 \mathrm{~lm}$ in length, and $200 \mathrm{~nm}$ in thickness, and attached to a soft magnetic nickel body on one side; authors demonstrated the forward motion of the structure in the direction of the helical axis by applying a rotational magnetic field in that direction and pointed out that linear swimming velocity was affected not only by the size of the magnetic head, but also the strength of the applied magnetic field. Shahnaz Bahmanyar et al. [20] Double Helices Propulsion Mechanism consists of two parallel helices with a single axis rotating in the same direction. The outer helix acts as the main propulsion component, and the inner helix, which is made of a Shape
Memory Alloy (SMA), controls the forward velocity during swimming. This mechanism, by varying the geometrical parameters of its helical tail, can change the forward velocity of the helical swimmer robot that is required by its predefined missions. In order to study the effects of geometric parameters on the forward velocity in the single helical swimmer, a hydrodynamic model based on Slender Body Theory (SBT) is implemented.

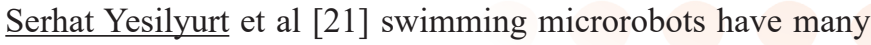
advantages for biomedical applications such as targeted drug delivery, simple surgical tasks including opening of clogged arteries and as diagnostic tools. In this swimming of microrobots is examined in water and glycerin filled channels. Propulsion of microrobots is enabled by means of an external magnetic field that rotates in the axial direction of the channel and forces robots to rotate about the axis of the helical tail. Rotation of the helical tail resulted in a screw-like motion of the robot reaching speeds up to several millimeters per second for a 2-mm long robot. Kenta Ishimoto and Darren G. Crowdy et al [22] induction of flow is commonly used to control the migration of a microswimmer in a confined system such as a microchannel. The motion of a swimmer, in general, is governed by nonlinear equations due to non-trivial hydrodynamic interactions between the flow and the swimmer near a wall. Peter Mueller and Jean-Luc Thiffeault et al [23] The model captures important aspects of real microswimmers such as a time-dependent flagellar motion and a no-slip body. The model consists of a rigid sphere for the body and a time-dependent moving Stokeslet representing the flagella. Hakan Ceylan, I. Ceren Yasa, Oncay Yasa, A. Fatih Tabak, Joshua Giltinan, Metin Sitti* et al [24] An ideal material solution should convey the idea of short-term inertness in the body while a microrobot should be degradable with the lowest possible waste profile in the long-term. Here, we designed, explored, and characterized in vitro a hydrogel based biodegradable helical microswimmer remotely controlled by rotating magnetic fields. The use of a biopolymer derivative, gelatin methacryloyl, to make microrobots will pave the way for making patient-specific microrobots using their own biomaterials. DNA-based selfassembly can serve as a general and flexible tool to construct artificial flagella of several micrometers in length and only tens of nanometers in diameter. By attaching the DNA flagella to biocompatible magnetic microparticles, we provide a proof of concept demonstration of hybrid structures that, when rotated in an external magnetic field, propel by means of a flagellar bundle, similar to self-propelling peritrichous bacteria [25]. Our theoretical analysis predicts that flagellar bundles that possess a length-dependent bending stiffness should exhibit a superior swimming speed compared to swimmers with a single appendage.

DeGong $^{\text {ab }}$ JunCai ${ }^{\text {ac }}$ NuoerCeli $^{\mathrm{a}}$ LinFeng $^{\mathrm{a}}$ YonggangJiang ${ }^{\mathrm{a}}$ DeyuanZhangac et al [26] Bio-inspired magnetic helical microswimmers have attracted much attention for performing complex tasks at low Reynolds numbers. To fabricate such helical microswimmers with enhanced propulsion velocity in bulk, Spirulina were electroless plated with nickel coating. 
The morphology, coating composition and magnetic property of the as-prepared microswimmers were investigated in detail. The results showed that homogeneous and compact nickel coating was successfully deposited on the helical biotemplates with good surface quality. Tiantian $\mathrm{Xu}$, Gilgueng Hwang, Nicolas Andreff, Stéphane Régnier et al [27] we proposed a scaled-up experimental system for preliminary analyses of helical swimmers swimming abilities. This scaled-up system consists in a rotating permanent magnet driving scaled-up helical microswimmers. A helical swimmer with a magnetic square head and a non magnetic tail showed a cut-off frequency with gentle decline. Its rotation frequency was about half of the frequency of the rotating field until it reached its maximum, and then its rotation frequency decreased to zero. A helical swimmer with its helical tail uniformly coated by ferromagnetic material showed a saturation of frequency. Its rotation frequency increased synchronously with the rotating magnetic field, then it stabilized at a value slightly smaller than its maximum. T. Morita, T. Omori, and T. Ishikawa, Phys. Rev. E 98, 023108 (2018) et al [28] a microcapsule swimmer that underwent amoeboidlike shape deformation under vertical fluid oscillation and showed the advantages of using a solid membrane and fluid oscillation in terms of swimmer controllability. Although the microcapsule was capable of migrating in the Stokes flow regime, the propulsion direction was limited to vertically upward or downward. The horizontal propulsion can be understood in terms of effective and recovery strokes, i.e., a stroke swimmer, whereas vertical propulsion is similar to rigid body motion induced by a torque, i.e., a torque swimmer. A M Leshansky1,4, O Kenneth2, O Gat3 and J E Avron2 et al [29] the self-locomotion of an elongated microswimmer by virtue of unidirectional tangential surface treadmilling. We show that the propulsion could be almost frictionless, as the microswimmer is propelled forward with the speed of the backward surface motion, i.e. it moves through an almost quiescent fluid. This swimming technique used the special spheroidal coordinates and also finds an explicit closed-form optimal solution for a two-dimensional treadmiller via complex-variable techniques.

Annalisa Quaini and Suncica Canic et al [30] the swimmers are assumed to be made of elastic cylindrical hollow tubes. The swimming is generated by the contractions of the tube's elastic membrane walls producing a traveling wave in the form of a "step-function" traversing the swimmer from right to left, propelling the swimmer from left to right. The problem is motivated by medical applications such as drug delivery. The influence of several non-dimensional design parameters on the velocity of the swimmer is investigated, including the swimmer aspect ratio, and the amplitude of the traveling wave relative to the swimmer radius. An immersed boundary method based on a finite element method approach is successfully combined with an elastic spring network model to simulate the two-way fluidstructure interaction coupling between the elastic cylindrical tube and the flow of a 3D viscous, incompressible fluid.

Modelling and Analysis

The design of all micro swimmers is done by using the
Solidworks software. Here, three different geometric models is designed in the Solidworks. The standard values for a cylindrical head is shown in table 1 .

\begin{tabular}{|l|l|l|l|}
\hline Symbol & Description & Values $(\mu \mathrm{m})$ & $\begin{array}{l}\text { Non- } \\
\text { Dimensional } \\
\text { values }\end{array}$ \\
\hline Dh & $\begin{array}{l}\text { Diameter of the } \\
\text { cylindrical head }\end{array}$ & 400 & 1 \\
\hline Lh & $\begin{array}{l}\text { Length of the } \\
\text { cylindrical head }\end{array}$ & 600 & 1.500 \\
\hline Wave length of the & 625 & 1.5625 \\
\hline tail & Wave amplitude & 200 & 0.5 \\
\hline Lt & Length of the tail & 1250 & 3.125 \\
\hline Dw & Wire diameter & 130 & 0.325 \\
\hline Ls & $\begin{array}{l}\text { Total length of the } \\
\text { micro swimmer }\end{array}$ & 1850 & 4.265 \\
\hline Dch & $\begin{array}{l}\text { Length of the } \\
\text { channel }\end{array}$ & 1000 & 2.5 \\
\hline $\mathrm{N} \lambda$ & Number of waves & 2 & 2 \\
\hline
\end{tabular}

Table 1: Standard values of Cylindrical Headed Micro swimmer

Here the values are for a cylindrical head, by taking the diameter of the cylinder we designed the geometry of sphere. The dimensions are in micro order because of such micro design. These values are taken from the reference paper where they experimented the desired design practically in fluid medium.

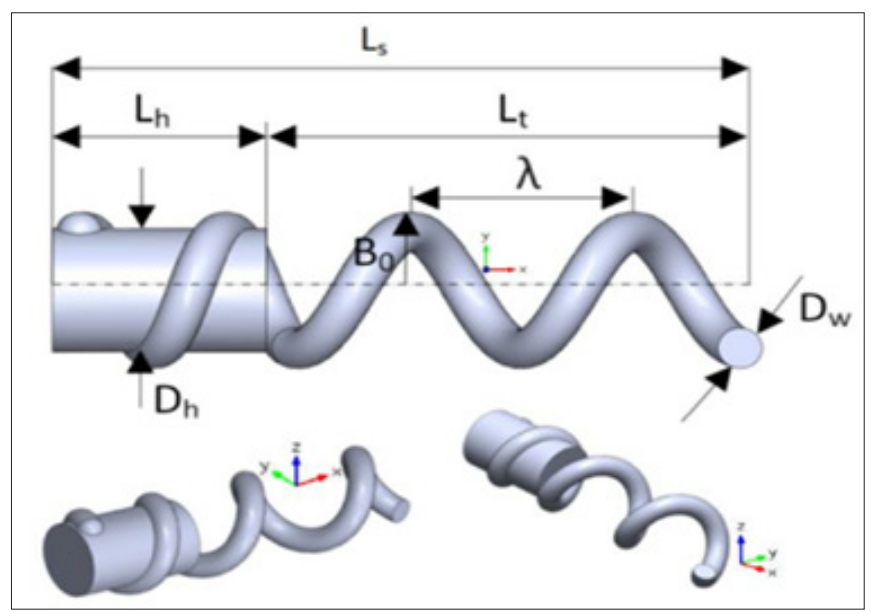

Figure 4 : Cylindrical head with a helical tail

After completing design of all the three heads, we have to design a helical shaped tail with one end attached to the head of the micro-swimmer. 


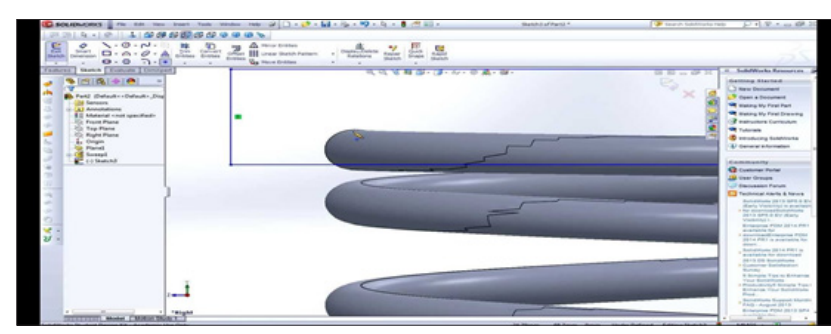

Figure 5: Spiral Curve

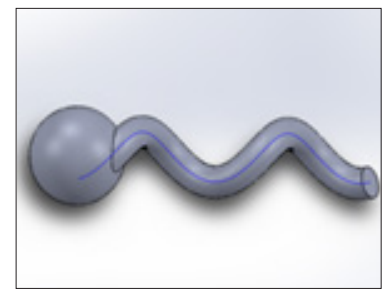

Figure 6: Spherical Head

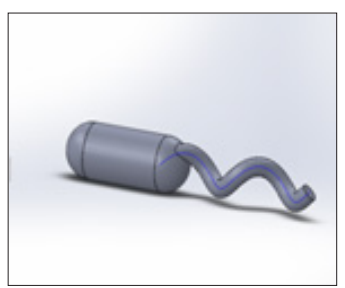

Figure 7: Capsule Head

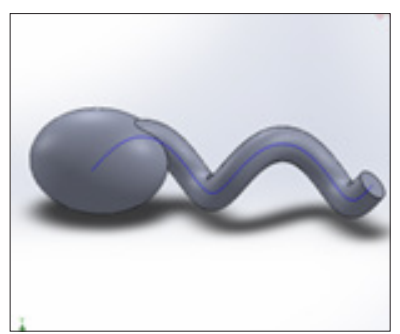

Figure 8: Elliptical Head

This helical tail has to be attached to those three different heads by using the assembly option. After assembling the tail to these heads, the figures 6-8 are modelled using Solidworks. Then, create a domain to send these bodies into it and analyzing the hydrodynamic characteristics of bodies when flow through the blood. Consider the domain into which the body has to be send. This domain may takes the geometry of the largest of the arteries. Its name is Aorta. The domain is also designed by using the solidworks software. The design dimensions of the domain are: Ascending aorta- length $-160 \mathrm{~mm}$, diameter- $2.1 \mathrm{~cm} 2$ and Descending aorta- length-290mm, diameter- $1.6 \mathrm{~cm} 2$. The aorta is the largest artery in the body. The aorta begins at the top of the left ventricle, the heart's muscular pumping chamber. The heart pumps blood from the left ventricle into the aorta through the aorticvalve. One way of classifying a part of the aorta is by anatomical compartment, where the thoracic aorta (or thoracic portion of the aorta) runs from the heart to the diaphragm. The aorta then continues downward as the abdominal aorta (or abdominal portion of the aorta) diaphragm to the aortic bifurcation.

Another system divides the aorta with respect to its course and the direction of blood flow. In this system, the aorta starts as the ascending aorta then travels superiorly from the heart and then makes a hairpin turn known as the aortic arch. Following the aortic arch, the aorta then travels inferiorly as the descending aorta. The descending aorta has two parts. The aorta begins to descend in the thoracic cavity, and consequently is known as the thoracic aorta. After the aorta passes through the diaphragm, it is known as the abdominal aorta. The aorta ends by dividing into two major blood vessels, the common iliac arteries and a smaller midline vessel, the median sacral artery.

The ascending aorta begins at the opening of the aortic valve in the left ventricle of the heart. It runs through a common pericardial sheath with the pulmonary trunk. These two blood vessels twist around each other, causing the aorta to start out posterior to the pulmonary trunk, but end by twisting to its right and anterior side. The transition from ascending aorta to aortic arch is at the pericardial reflection on the aorta. The descending aorta is part of the aorta, the largest artery in the body. The descending aorta begins at the aortic arch and runs down through the chest and abdomen. The descending aorta anatomically consists of two portions or segments, the thoracic and the abdominal aorta, in correspondence with the two great cavities of the trunk in which it is situated. Within the abdomen, the descending aorta branches into the two common iliac arteries which serve the pelvis and eventually legs and shown in figure 9.

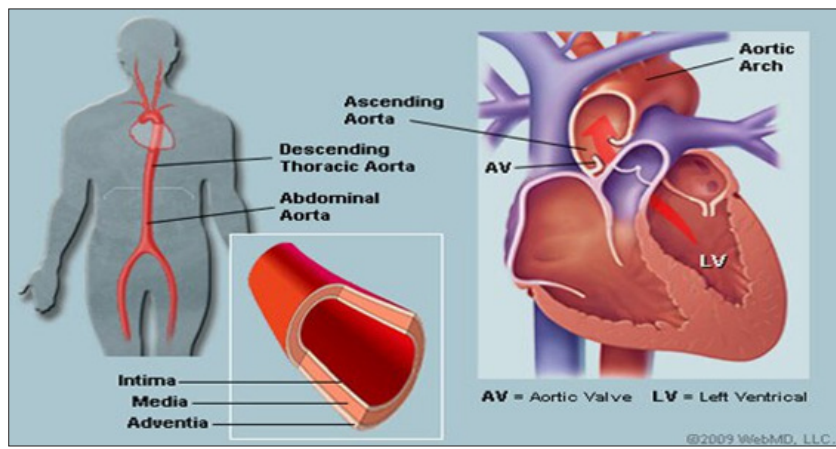

Figure 9: Aorta

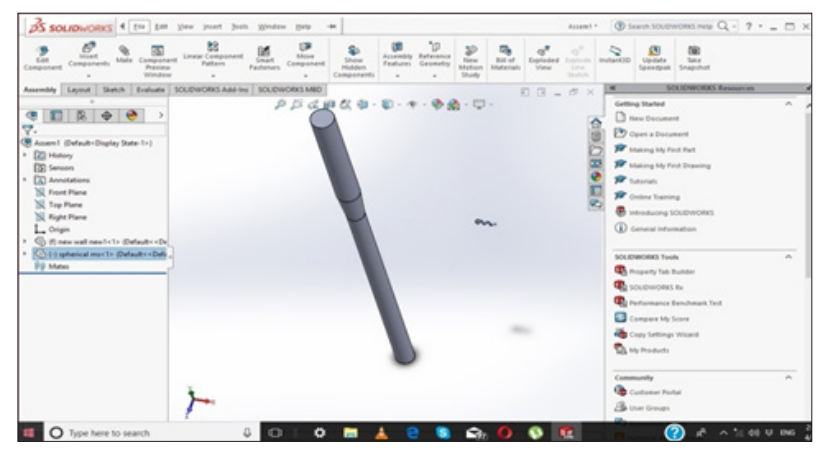

Figure 10: Assembly of domain and microswimmer

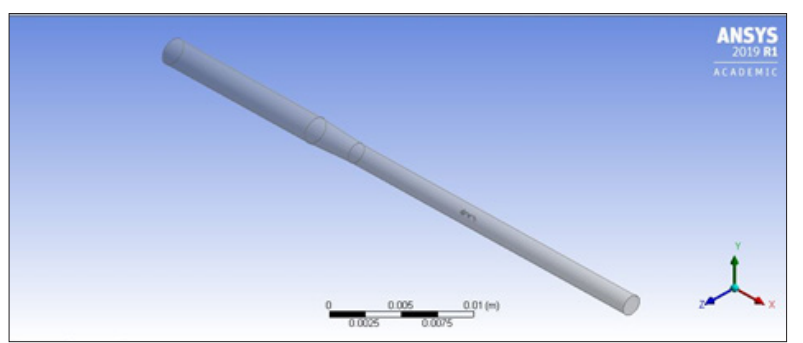

Figure 11: Domain with the spherical head microswimmer

The assembly that obtained is to be in IGS file format so as to move or can import for further analysis procedure. Before 
starting the analysis in the Ansys Workbench and had to select the fluent fluid flow from the analysis systems on the left side of the workbench.

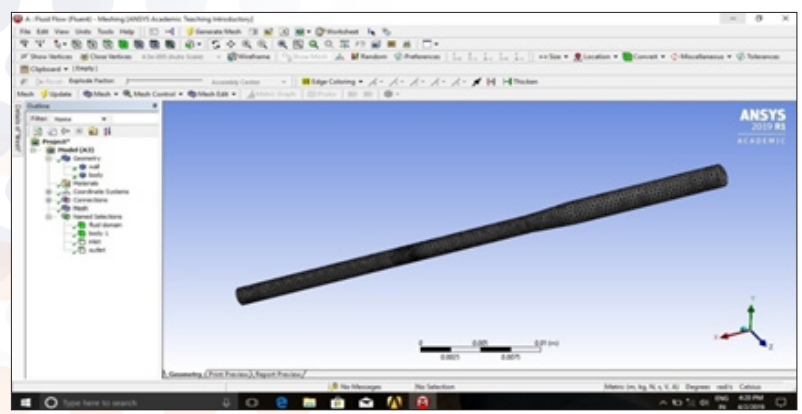

Figure 12 : Meshing view

The models, materials, cell zone conditions, and the boundary conditions are given and select models option and put on the energy option and select viscous, laminar, and k-epsilon from them. The materials should be given here, Here we taken blood as the fluid and solid iron oxide as body and Blood properties are given as follows in table 2 .

\begin{tabular}{|l|l|}
\hline PROPERTIES & STANDARD VALUES \\
\hline Density & $1060 \mathrm{Kg} /$ cubic-metre \\
\hline Specific gravity & $3513 \mathrm{~J} / \mathrm{Kg} . \mathrm{K}$ \\
\hline Thermal conductivity & $0.52 \mathrm{~W} / \mathrm{m} . \mathrm{K}$ \\
\hline Viscosity & $0.003 \mathrm{Kg} / \mathrm{m}-\mathrm{s}$ \\
\hline
\end{tabular}

Table 2: Blood Properties

The Cell zone conditions; After inserting the materials, the body has given iron oxide element based on literature survey and domain is filled with blood and have given the standard values for the blood from the references. Boundary conditions are considered. In this, the inlet velocity and the outlet pressure are given to the domain. Inlet velocity- $0.66 \mathrm{~m} / \mathrm{sec}$ and Outlet pressure- $11000 \mathrm{~Pa}$. The Solution initialization are hybrid initialization and standard initialization.

ANSYS Fluent will compute and update the Initial Values based on the conditions defined at all boundary zones. If you want to change one or more of the values, you can enter new values manually in the fields next to the appropriate variables. The run calculations are shown in figure 13. Here we have given 100 iterations to calculate the convergence of the solution, but we got the convergent solution at 50 iterations. By taking velocity on $y$-axis and position which is default on $\mathrm{x}$-axis, we generated the graph.

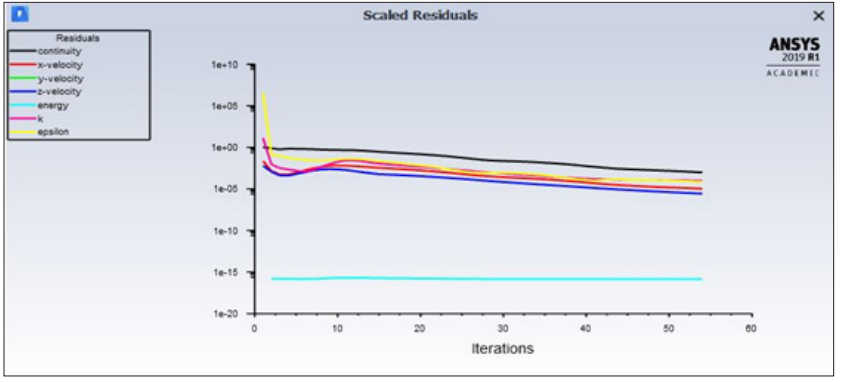

Figure 13: Iterations view

\section{Results \& Discussions}

From this analysis, the graphs of velocity and positions are plotted and studied different head microswimmers, the trends of velocity magnitude differs a lot. Blood is the fluid used for the analysis of these variations as our main concern is all about the Biomedical applications like drug delivery. Here had a domain and blood is flowing in it and the micro-swimmer moves against the flow. At that instance the blood try to push the swimmer along with its flow. There creates some Reaction force. Due to this the velocity differ at each position of the body in the domain. The graphs are plotted, taking position on $\mathrm{x}$-axis and velocity magnitude on y axis. The results of spherical headed micro swimmer are shown below from figures 14-18 and Capsule type headed micro swimmer is from 19-22 and Ellipitical headed type micro swimmer is from figures 23-26.

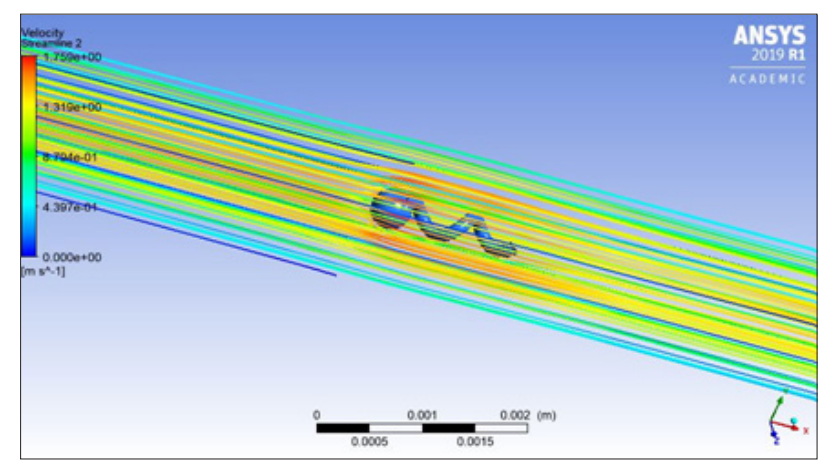

Figure 14: streamlines

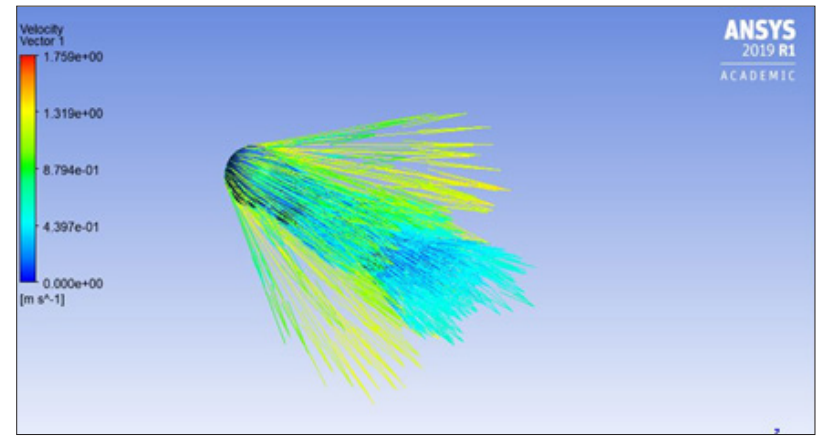

Figure 14: velocity vector 


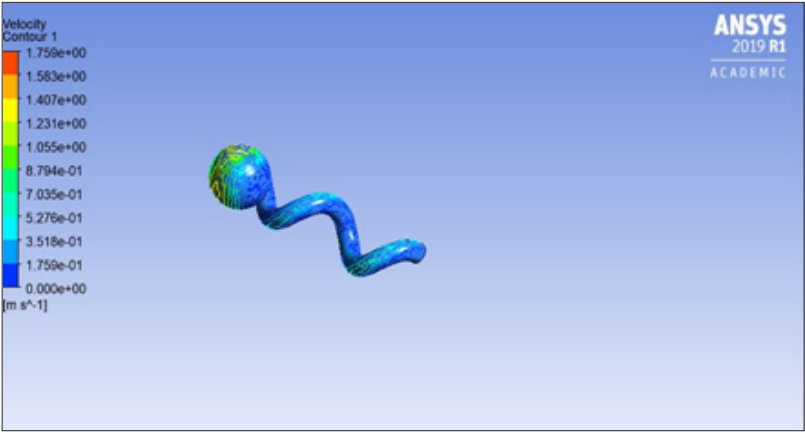

Figure16: velocity contour

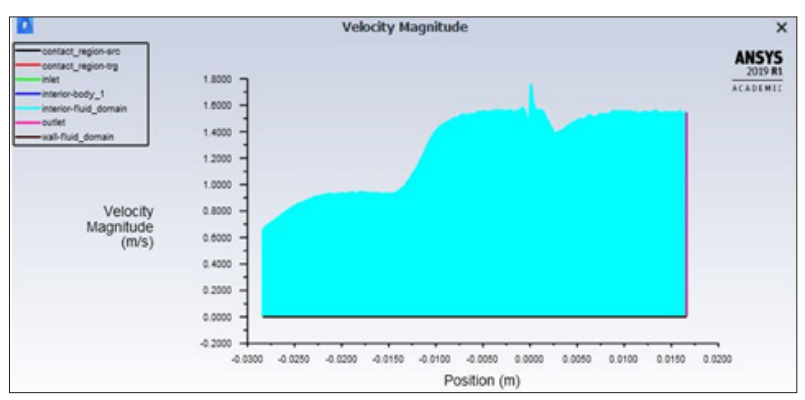

Figure 17: $x-y$ plot in Ansys

$\mathrm{X}$-axis - position (m)

$\mathrm{Y}$-axis - velocity magnitude $(\mathrm{m} / \mathrm{s})$

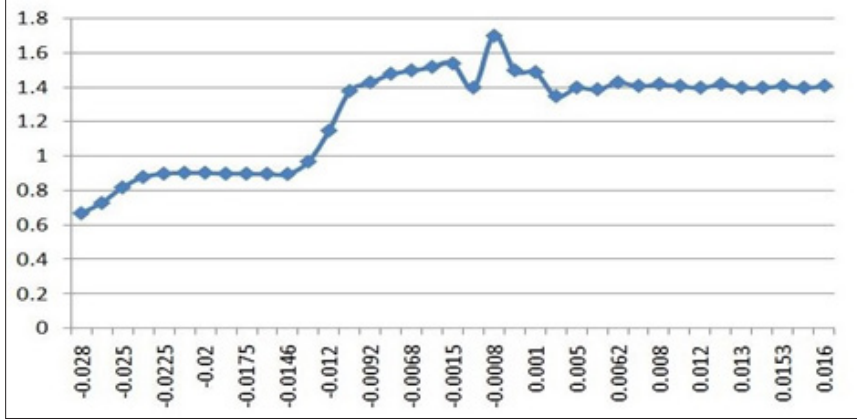

Figure 18 : X-Y Plot

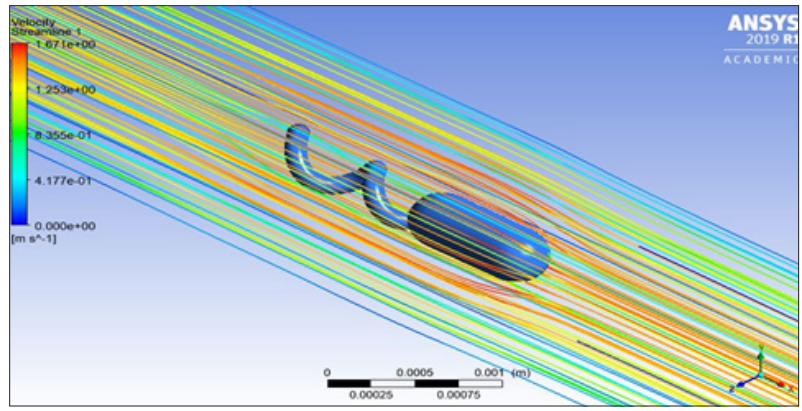

Figure 19 : stream lines

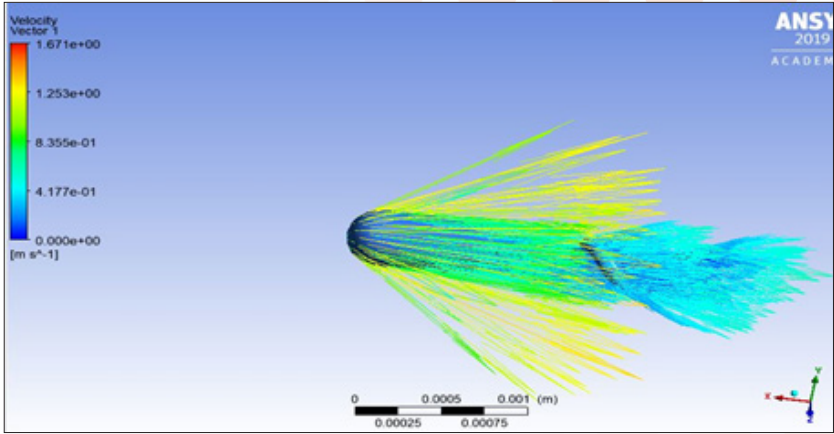

Figure 20 : velocity vector

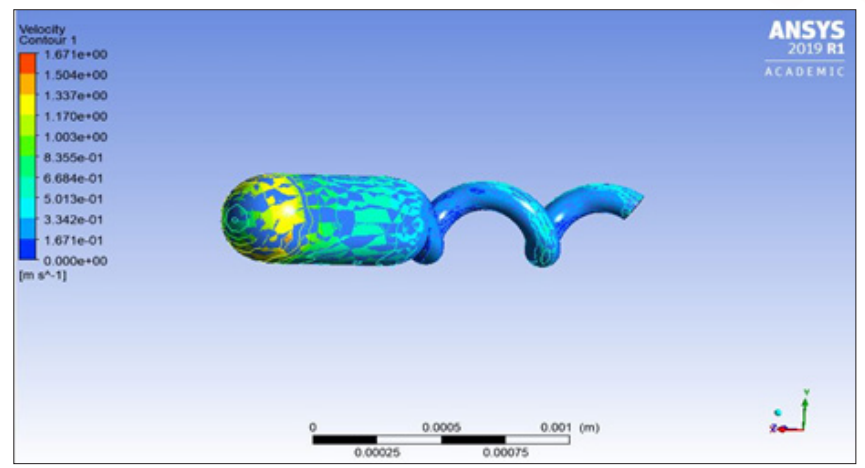

Figure 21 : velocity contour

$\mathrm{X}$-axis - position (m)

$\mathrm{Y}$-axis - velocity magnitude $(\mathrm{m} / \mathrm{s})$

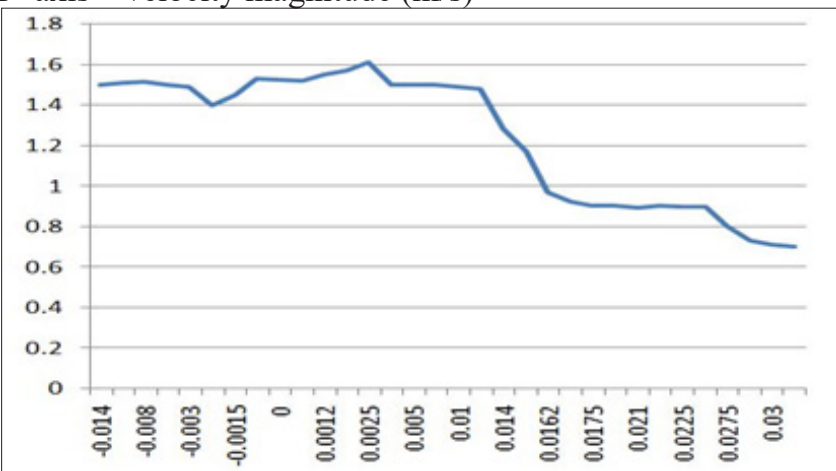

Figure 22 : X-Y Plot

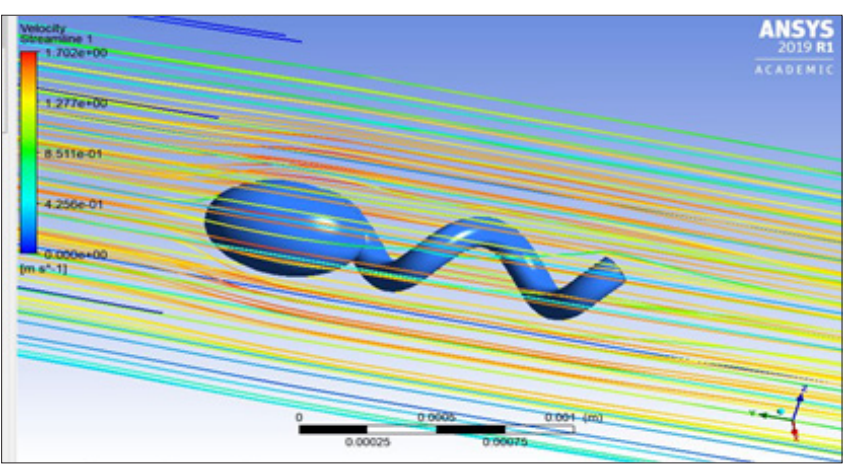

Figure 23 : streamlines 


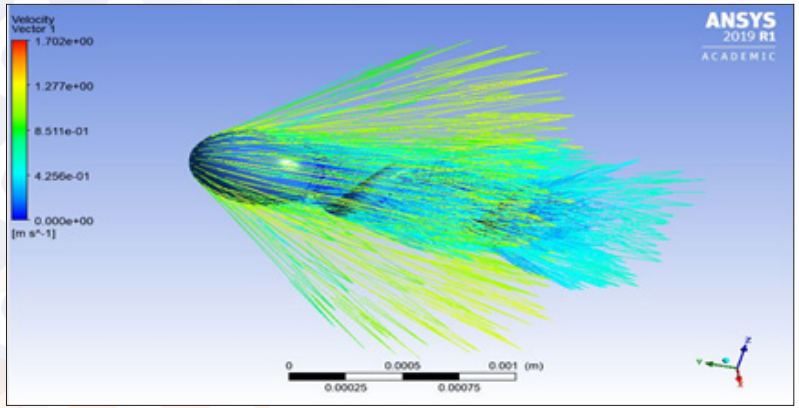

Figure 24: velocity vector

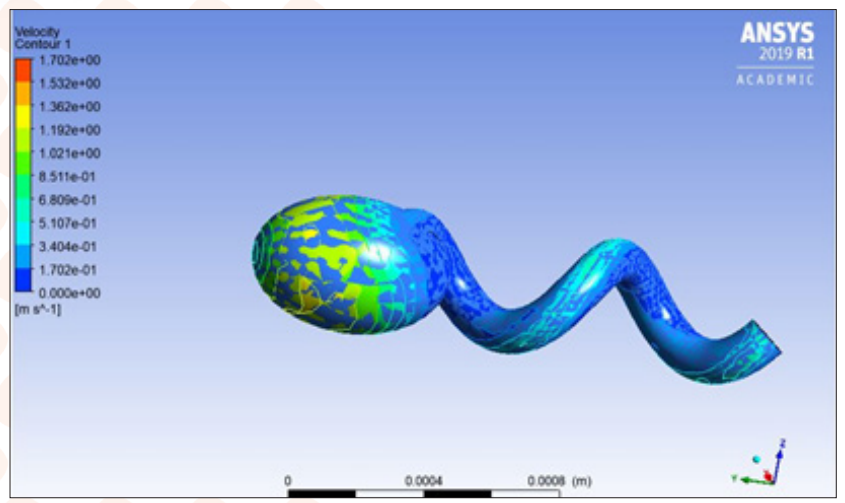

Figure 25: velocity vector

$\mathrm{X}$-axis - position $(\mathrm{m})$

$\mathrm{Y}$-axis - velocity magnitude $(\mathrm{m} / \mathrm{s})$

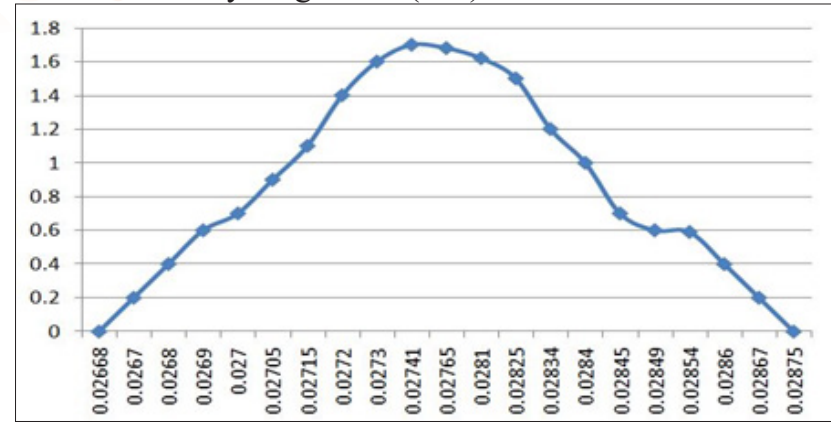

Figure 26: velocity vector

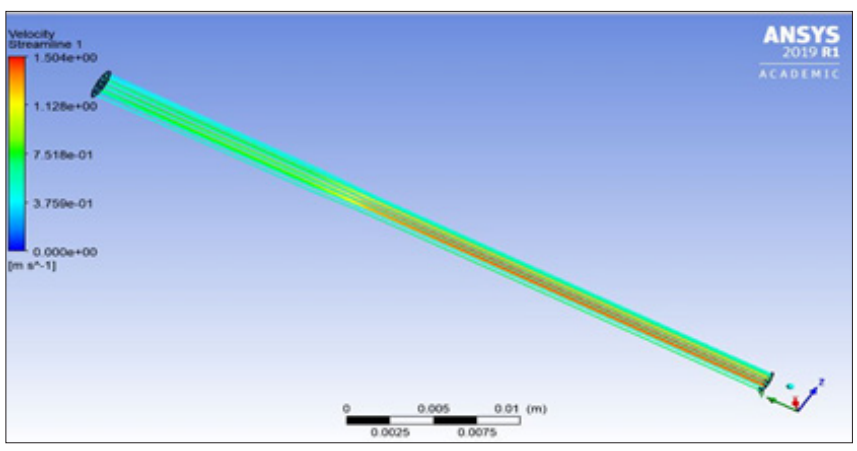

Figure 27: streamlines
X-axis- position (m);

Y-axis- velocity magnitude $(\mathrm{m} / \mathrm{s})$

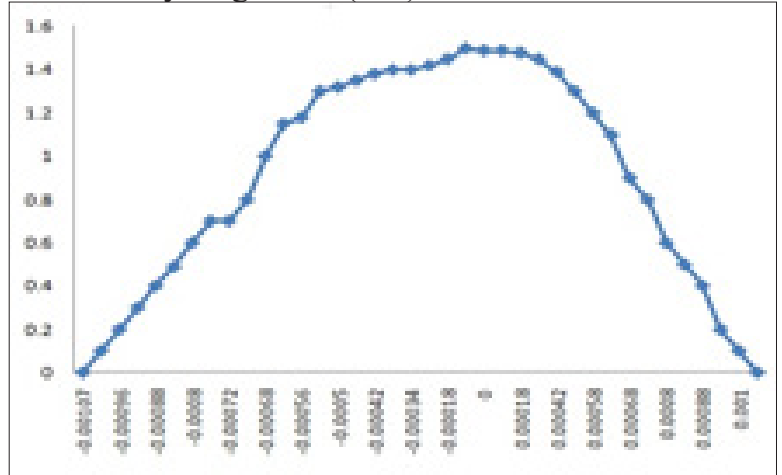

Figure 28: X-Y Plot for Domain

The above graphs showing the streamlines, velocity vectors and velocity gradients of three different heads and also for domain that was shown from figures 27-28. But, don't know whether which model is best for the application,compared to others. For this, had taken domain with no body. Then, compared these reference graphs with the graphs of three models. The graphs of elliptical head are somewhat close to the reference graphs. So we conclude that it would be the best.

\section{Conclusion}

The following conclusions are framed from the research work:

- The considered three models of micro-swimmers were taken from the standard journals and designed and developed in SOLIDWORKS. The head and tails are created as a single component based on the required dimensions. Then the part file is converted into IGS file to open the file in ANSYS software.

- Analysis and flow simulation is done in ANSYS FLUENT under necessary conditions, by creating a domain for the micro-swimmer. The graphs are presented between velocity and positioning of micro-swimmer.

- Then these graphs are considered as results, whose are the reactions between the blood and micro-swimmer.

- When the blood is continuously flowing in the aorta, the swimmer will be injected into it. The swimmer initially is in rest before the activation of its mechanism for few milliseconds. In this small time lapse, the blood flow strikes the swimmer head. In this instance, the velocity magnitude with respect to position are plotted.

- Finally, comparision is done between the three different models on the basis of their velocity variations. 


\section{References}

1. Gray and Hancock et al. "The propulsion by large amplitude waves of uniflagellar micro-organisms of finite length".

2. Berke AP, Turner L, Berg HC, Lauga E. "Hydrodynamic attraction of swimming microorganisms by surfaces".

3. Najafi et al. "Mini and Micro Propulsion for Medical Swimmers".

4. Jonathan R. Howse. "Self-motile colloidal particles: from directed propulsion to random walk".

5. Martel S., Mohammadi M., Felfoul O., Lu Z., and Pouponneau P., "Flagellated magneto tactic bacteria as controlled MRI-trackable propulsion and steering systems for medical nanorobots operating in the human microvasculature", Int. J. Robot. Res., vol. 28, pp. 571582, 2009

6. Bradely j.Nelson et al. Wireless Intraocular Microrobots: Opportunities and Challenges

7. Li Zhang,1 Jake J. Abbott. "Artificial bacterial flagella: Fabrication and magnetic control".

8. Andrew D. Gilbert "Theory of ferromagnetic materials".

9. Eric Lauga, Willow R. DiLuzio. "Swimming in Circles: Motion of Bacteria near Solid Boundaries".

10. Bernard Bonnard et al. "Sub-Riemannian geometry, Hamiltonian dynamics, micro-swimmers, copepod nauplii and copepod robot".

11. Francisco Alarc'on and Ignacio Pagonabarraga. "Micro-swimmer suspensions using high performance computing"..

12. Joost de Graaf et al. "Lattice-Boltzmann Simulations of Microswimmer-Tracer Interactions".

13. Adam Wysocki et al. "Dynamics of swimming bacteria at complex interfaces".

14. Hancock, G.J., "The self-propulsion of microscopic organisms through liquids", Proceedings of the Royal Society of London, Series A, Vol. 217, No. 1128, pp. 96121, 1953.

15. Kathrin E. Peyer et al. " Microbiorobotics: Biologically Inspired Microscale Robotic Systems".

16. J E Avron et al. "A frictionless microswimmer".

17. Dr. Ignacio Pagonabarraga et al "Fluid-Structure Interactions in Soft-Matter Systems: From the Mesoscale to the Macroscale".

18. J E Avron, O Kenneth and D H Oaknin " Pushmepullyou: an efficient micro-swimmer".

19. Martel et al. "Simulation-based analysis of micro-robots swimming at the center and near the wall of circular minichannels".

20. Shahnaz Bahmanyar., "Development of a new mechanism to change velocity in helical swimmer robot at low Reynolds Number".

21. Serhat Yesilyurt- Magnetically actuated micro swimming of bio-inspired robots in mini channels.

22. Kenta Ishimoto and Darren G. Crowdy "Dynamics of a treadmilling microswimmer near a no slip wall in simple shear."

23. Peter Mueller and Jean-Luc Thiffeault "Fluid transport and mixing by an unsteady microswimmer."

24. Hakan Ceylan, I. Ceren Yasa, Oncay Yasa, A. Fatih Tabak, Joshua Giltinan, Metin Sitti “3D-Printed Biodegradable Microswimmer for Drug Delivery and Targeted Cell Labeling."

25. Alexander M. Maier, Cornelius Weig, Peter Oswald, Erwin Frey, Peer Fischer, and Tim Liedl "Magnetic Propulsion of Microswimmers with DNA-Based Flagellar Bundles."

26. DeGong, JunCai NuoerCeli, LinFeng, YonggangJiang, DeyuanZhang "Bio-inspired magnetic helical microswimmers made of nickel-plated Spirulina with enhanced propulsion velocity."

27. Tiantian $\mathrm{Xu}$, Gilgueng Hwang, Nicolas Andreff, Stéphane Régnier "27Modeling and Swimming Property Characterizations of Scaled-Up Helical Microswimmers."

28. Takeru Morita, Toshihiro Omori, and Takuji Ishikawa "Biaxial fluid oscillations can propel a microcapsule swimmer in an arbitrary direction."

29. A M Leshansky1,4, O Kenneth2, O Gat3 and J E Avron2 1 Department of Chemical Engineering Technion, Haifa, 32000, Israel "29A frictionless microswimmer."

30. Annalisa Quaini and Suncica Canic "A study of selfpropelled elastic cylindrical micro-swimmers using modeling and computation."
Copyright: C2021 Roopsandeep Bammidi. This is an open-access article distributed under the terms of the Creative Commons Attribution License, which permits unrestricted use, distribution, and reproduction in anymedium, provided the original author and source are credited. 